\title{
Comunicación
}

\section{Evidencia hematológica de dismegacariocitopoyesis selectiva en un perro con ehrlichiosis canina}

\author{
Hematological evidence of selective dysmegakaryocytopoiesis in a dog \\ with canine ehrlichiosis \\ Alvaro Vasquez Y., ${ }^{1,3}$, Olga Li E. ${ }^{1}$, Miguel Cervantes S. ${ }^{1}$, Deysi Masgo C. ${ }^{1}$, \\ Iran Zavaleta C. ${ }^{\text {, }}$ Luis Hoyos S. ${ }^{1,2}$
}

\section{Resumen}

Se describe el caso de una perra Ovejero Inglés de dos años que fue llevada a consulta a la Clínica de Animales Menores de la Facultad de Medicina Veterinaria de la Universidad Nacional Mayor de San Marcos por presentar letargia reciente. Los exámenes sanguíneos en la paciente revelaron pancitopenia, presencia de mórulas compatibles con Ehrlichia spp en leucocitos, disproteinemia y seropositividad frente a E. canis / E. ewingii. Se pudo observar en los extendidos de Buffy coat la presencia de múltiples megacariocitos displásicos, los cuales exhibieron alteraciones tales como asincronía de maduración nuclear, núcleos no lobulados a hipolobulados, aspecto pseudoarticulado del núcleo y emperipolesis megacariocitica de neutrófilos, sin observarse alteraciones mielodisplásicas en los restantes linajes celulares. Las características descritas en su conjunto representan la primera evidencia hematológica de dismegacariocitopoyesis selectiva en un perro con ehrlichiosis canina en el Perú.

Palabras clave: buffy coat, dismegacariocitopoyesis, emperipolesis, Ehrlichia spp

\section{AbSTRACT}

The case of a two-year-old English sheep bitch that was taken to the Small Animal Clinic of the Faculty of Veterinary Medicine, Universidad Nacional Mayor de San Marcos for presenting recent lethargy is described. Blood tests revealed pancytopenia, presence of morulae compatible with Ehrlichia spp in leukocytes, dysproteinaemia and

\footnotetext{
${ }^{1}$ Laboratorio de Patología Clínica Veterinaria, Facultad de Medicina Veterinaria, Universidad Nacional Mayor de San Marcos, Lima, Perú

${ }^{2}$ E-mail:_luis.hoyos@unmsm.edu.pe

${ }^{3}$ E-mail: alvaro.vasquez5@unmsm.edu.pe
}

Recibido: 18 de febrero de 2019

Aceptado para publicación: 8 de enero de 2020

Publicado: 31 de marzo de 2020 
seropositivity against E. canis / E. ewingii. The presence of multiple dysplastic megakaryocytes, which exhibited alterations such as asynchrony of nuclear maturation, nuclei not lobed to hypolobulated, pseudo-articulated aspect of the nucleus and megakaryocytic emperipolesis of neutrophils were observed, without myelodysplastic alterations in the remaining cell lineages. The characteristics described represent the first haematological evidence of selective dysmegacariocytopoiesis in a dog with canine ehrlichiosis in Peru.

Key words: buffy coat, dysmegakaryocytopoiesis, emperipolesis, Ehrlichia spp

\section{INTRODUCCIÓN}

La ehrlichiosis canina es una enfermedad transmitida por garrapatas a perros y presente a nivel global. El agente causal es Ehrlichia spp, bacteria intracelular obligada, gramnegativa, de aspecto redondo a pleomórfico, que se caracteriza por infectar a leucocitos (polimorfonucleares y mononucleares). Los perros son susceptibles a la infección por E. canis, E. chaffeensis y $E$. ewingii (Rikihisa, 1991).

La médula ósea puede ser blanco de múltiples patógenos transmitidos por vectores, incluyendo a Ehrlichia spp, causando alteraciones sustanciales en el número y función de los eritrocitos, granulocitos, monocitos, linfocitos y megacariocitos (De Tommasi et al., 2014). Los síndromes mielodisplásicos (SMD) representan un grupo de condiciones caracterizadas por la morfología anormal de una o más de las líneas celulares en médula ósea, y suelen manifestarse con anemia de tipo no regenerativa, leucopenia y trombocitopenia (Tefferi y Vardiman, 2009).

La dismegacariocitopoyesis es un tipo de SMD, el cual es definido por la Organización Mundial de la Salud (OMS) como una displasia de más del $10 \%$ del total de megacariocitos en el extendido de médula ósea de un paciente (Swerdlow et al., 2017). La dismegacariopoyesis está usualmente asociada con otros SMD, pudiendo presentarse en forma simultánea con diseritropoyesis y disgranulopoyesis. Por consiguiente, reportes de la ocurrencia de dismegacariocitopoyesis como una entidad separada, medicamente conocida como dismegacariocitopoyesis selectiva, es rara en la literatura médica, tanto humana como veterinaria (Weiss, 2004).

Los SMD, incluyendo a la dismegacariocitopoyesis, pueden ser clasificados con base al agente causal. Los SMD primarios pueden ser idiopáticos o por mutaciones adquiridas en uno o más linajes de las células madre hematopoyéticas (Weiss y Smith, 2000). Las causas secundarias de los SMD son asociadas con la administración de quimioterapéuticos, estrógenos o cloranfenicol, exposición a toxinas, infecciones parasitarias o virales, enfermedades inmunomediadas, exposición a radiaciones y deficiencia de hierro (Weiss y Aird, 2001; Weiss, 2005). En perros, los SMD secundarios como consecuencia de enfermedades inmunomediadas han sido relacionados con anemias hemolíticas inmunomediadas y trombocitopenias inmunomediadas (Weiss y Aird, 2001). También se ha reportado que se producen como resultado de la infección por patógenos transmitidos por vectores (Babesia vogeli, Anaplasma platys, Leishmania infantum y Hepatozoon canis) (De Tommasi et al., 2014).

Entre las anormalidades morfológicas observadas en los megacariocitos displásicos se incluye la presencia de micromegacariocitos $(<15 \mu \mathrm{m})$, núcleos no lobulados o hipolobulados, asincronía de maduración nu- 
Cuadro 1. Resultados de los exámenes hematológicos en una perra Ovejera Inglés nulípara de dos años

\begin{tabular}{|c|c|c|}
\hline Parámetro $^{1}$ & $\begin{array}{c}\text { Valor } \\
\text { absoluto }\end{array}$ & $\begin{array}{c}\text { Rangos de } \\
\text { valores }^{2}\end{array}$ \\
\hline $\begin{array}{l}\text { Eritrocitos } \\
\qquad\left(\mathrm{x} 10^{6} / \mu \mathrm{l}\right)\end{array}$ & 3.7 & $5-7$ \\
\hline $\begin{array}{l}\text { Hemoglobina } \\
\qquad(\mathrm{g} / \mathrm{dl})\end{array}$ & 7.6 & $12-18$ \\
\hline Hematocrito (\%) & 23 & $37-55$ \\
\hline $\begin{array}{l}\mathrm{VCM} \\
\text { (fl) }\end{array}$ & 62.2 & $66-77$ \\
\hline $\begin{array}{l}\text { CHCM } \\
\qquad(\mathrm{g} / \mathrm{dl})\end{array}$ & 33 & $32-36$ \\
\hline $\begin{array}{l}\text { Plaquetas } \\
\qquad\left(\times 10^{3} / \mu 1\right)\end{array}$ & 90 & $200-400$ \\
\hline $\begin{array}{l}\text { Leucocitos } \\
\qquad(\mu \mathrm{l})\end{array}$ & 4,900 & $\begin{array}{l}8,000- \\
13,000\end{array}$ \\
\hline $\begin{array}{l}\text { Abastonados } \\
\quad(\mu \mathrm{l})\end{array}$ & 98 & $0-390$ \\
\hline $\begin{array}{l}\text { Segmentados } \\
\quad(\mu 1)\end{array}$ & 4,263 & $\begin{array}{c}5,200- \\
9,750\end{array}$ \\
\hline $\begin{array}{l}\text { Linfocitos } \\
\qquad(\mu \mathrm{l})\end{array}$ & 539 & $\begin{array}{c}1,200- \\
3,900\end{array}$ \\
\hline \multicolumn{3}{|c|}{$\begin{array}{l}{ }^{1} \text { VCM: Volumen corpuscular medio; CHCM: } \\
\text { Concentración de hemoglobina corpuscular } \\
\text { media } \\
2 \text { Fuente: Laboratorio de Patología Clínica } \\
\text { Veterinaria, Universidad Nacional Mayor de } \\
\text { San Marcos }\end{array}$} \\
\hline
\end{tabular}

clear y evidencia de múltiples núcleos ampliamente separados (Weiss, 2004; Swerdlow et al., 2017). La detección prematura de los SMD secundarios resulta importante, pues los SMD representarían estados preleucémicos que podrían progresar en una leucemia mieloide aguda en un periodo indeterminado (Triantafyllidis et al., 2012). El presente caso representa la primera evidencia hematológica de dismegacariocitopoyesis selectiva secundaria en un perro con ehrlichiosis canina en el Perú.

\section{Caso Clínico}

Una perra Ovejera Inglés, nulípara, de dos años fue llevada a consulta a la Clínica de Animales Menores de la Facultad de Medicina Veterinaria de la Universidad Nacional Mayor de San Marcos (Lima, Perú), debido a que se mostraba decaída durante los últimos cinco días.

La dieta del animal consistía en alimento balanceado comercial para perros adultos. El propietario comentó que no observó garrapatas en su mascota, pero que el animal mantuvo contacto con animales infestados con ectoparásitos (pulgas y garrapatas). A la evaluación clínica, la paciente presentó 9.7 $\mathrm{kg}$ de peso corporal, temperatura rectal normal $\left(38.5^{\circ} \mathrm{C}\right)$, constantes fisiológicas dentro de los rangos normales y letargia (moderada a severa). No se detectó organomegalia ni linfoadenomegalia a la palpación.

Se colectaron muestras de sangre de la vena cefálica para realizar un hemograma completo y bioquímica sérica (Cuadro 1). Los frotis de sangre periférica fueron teñidos con tinción Wright de base acuosa. Para las observaciones citológicas se empleó un microscopio óptico de fondo claro Leica DM500® acoplado a una cámara Leica ICC50W ${ }^{\circledR}$ (Leica Microsystems, Alemania).

En los frotis de sangre periférica se observó moderada cantidad de linfocitos reactivos y linfocitos atípicos, leve anisocitosis eritroide y policromasia, moderada cantidad de plaquetas hipogranulares y macroplaquetas, así como la presencia de 1-2 estructuras intracitoplasmáticas entre 2 y $4 \mu \mathrm{m}$ de diámetro, redondeadas, de coloración grisácea, compatibles con mórulas de Ehrlichia spp en monocitos y neutrófilos.

En los extendidos de Buffy coat, evaluados en campo de alto poder $H P F(40 \mathrm{x})$ se apreciaron 1-2 estructuras celulares individuales compatibles con megacariocitos 


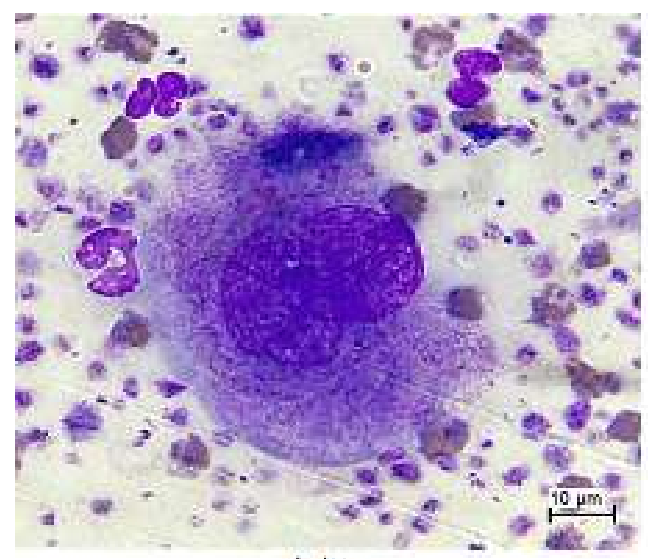

(a)

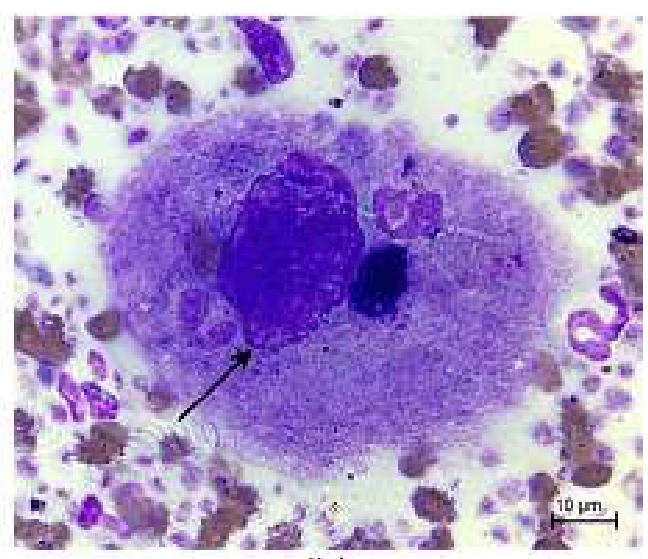

(b)

Figura 1. Frotis de sangre de una perra Ovejera Inglés nulípara de dos años. (a) Megacariocito displásico con bilobulación nuclear y escaso citoplasma granular. (b) Núcleo pseudoarticulado (flecha) de un megacariocito que evidencia asincronía de maduración nuclear. Buffy coat. Tinción Wright. 100x

displásicos, los cuales mostraron forma ovoide y diámetro $>50 \mu \mathrm{m}$. Dichas células exhibían en su mayoría una marcada asincronía de maduración nuclear, núcleos no lobulados o bilobulados con un patrón de cromatina granular grueso heterogéneo y un borde nuclear bien definido. Los nucleolos no fueron visibles. En el citoplasma se observó una fina granulación de coloración magenta de distribución difusa sobre un citoplasma ligeramente basófilo, la relación núcleo/citoplasma fue variable con tendencia a ser baja y los bordes citoplasmáticos fueron mal definidos (Figuras 1a, 1b). En algunos de los megacariocitos displásicos fue posible identificar la presencia intracitoplasmática de neutrófilos (Figura 2), fenómeno conocido como emperipolesis.

Se determinaron las proteínas totales y albúmina en suero $(\mathrm{g} / \mathrm{dl})$ mediante un método colorímetro (Wiener Lab., Argentina), a través de un analizador bioquímico semiautomático Photometer 5010 V5+ (Robert-Riele, Alemania). Los resultados se presentan en el Cuadro 2. Además, se realizó un descarte de patógenos transmitidos por vectores mediante el kit $\mathrm{SNAP}^{\circledR}{ }^{4}$ Dx ${ }^{\circledR}$ Plus (IDEXX

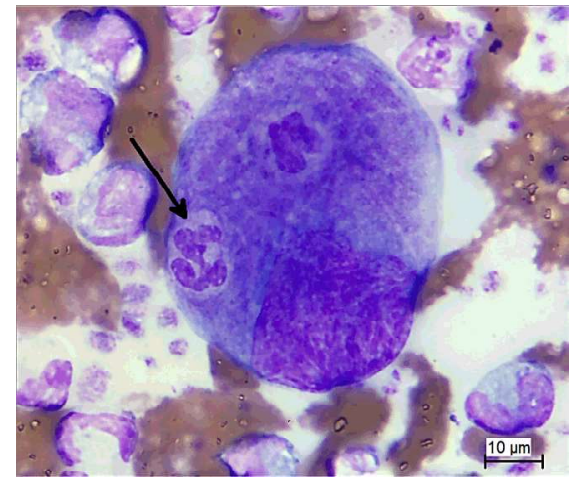

Figura 2. Emperipolesis megacariocítica de un neutrófilo (flecha). Buffy coat. Tinción Wright. 100x, en un frotis de sangre de una perra Ovejera Inglés nulípara de dos años

Laboratories, USA) encontrándose seropositividad para la detección de anticuerpos frente a E. canis / E. ewingii.

\section{Discusión}

Pese a que la dismegacariocitopoyesis selectiva secundaria es una rara condición mielodisplásica reportada en medicina vete- 
Cuadro 2. Cuantificación sérica de proteínas y albúmina de una perra Ovejera Inglés nulípara de dos años

\begin{tabular}{lcc}
\hline & Resultado & $\begin{array}{c}\text { Rangos de } \\
\text { valores }^{1}\end{array}$ \\
\hline Proteína $(\mathrm{g} / \mathrm{dl})$ & 6.1 & $5.5-7.5$ \\
Albúmina $(\mathrm{g} / \mathrm{dl})$ & 2.1 & $2.3-3.2$ \\
\hline${ }^{1}$ Fuente: Laboratorio de & Patología Clínica \\
Veterinaria, Universidad Nacional Mayor de \\
San Marcos
\end{tabular}

rinaria y humana, en el presente caso se reporta la presencia de megacariocitos displásicos en sangre periférica en un perro con ehrlichiosis canina, quien presentó mórulas compatibles con Ehrlichia spp en monocitos y neutrófilos, pancitopenia, hipoalbuminemia e hiperglobulinemia séricas y seropositividad frente a E. canis / E. ewingii.

Normalmente, los megacariocitos se caracterizan por presentar un núcleo multilobulado con 8-32 lóbulos fusionados, así como gránulos citoplasmáticos de coloración magenta que le imparten un aspecto rosa al citoplasma, siendo ello característico de los megacariocitos maduros (granulares). El diámetro oscila entre 50 y $200 \mu \mathrm{m}$, lo cual los hace fácilmente reconocibles en los extendidos debido a su abundante citoplasma (Latimer, 2011; Harvey, 2012). Sin embargo, los megacariocitos observados en el presente caso $(>50 \mu \mathrm{m})$ exhibieron una morfología atípica, y no fue posible distinguir la multiplicidad de lóbulos. Los megacariocitos fueron no lobulados o con hipolobulación nuclear (bilobulados) (Figuras 1a, 1b, 2). El núcleo en todos los casos mantuvo un aspecto «cerebriforme» o pseudoarticulado, debido a la existencia de marcadas indentaciones o convoluciones nucleares. Según Feng et al. (2016), dichas características displásicas en los megacariocitos podrían estar atribuidas a anormalidades en el proceso de endomitosis (lobulación nuclear).

Se podría presumir que los megacariocitos displásicos observados podrían provenir de la médula ósea, donde se produce la mayor megacariopoyesis, aunque también podrían provenir de otros órganos que realicen megacariopoyesis extramedular en perros, tales como el bazo, hígado y pulmones (Stockham y Scott, 2008). Sin embargo, no hubo evidencias clínico-laboratoriales que demuestren el compromiso de dichos órganos.

El fenómeno de la emperipolesis en megacariocitos representa un hallazgo infrecuente y suele ser asociado a patologías benignas y malignas. Este evento ha sido descrito en perros infectados con Hepatozoon canis (De Tommasi et al., 2014) y en humanos con linfomas, mielofibrosis, leucemias y síndromes mielodisplásicos (Schmitt et al., 2000). La emperipolesis, inclusión activa de una célula dentro de una célula huésped, se diferencia de la fagocitosis en que la célula engullida se puede mantener viable y con su estructura intacta, mientras que en la fagocitosis la célula envuelta es destruida por las enzimas lisosomales (Rastogi et al., 2014). Según Xia et al., (2008) la liberación de las proteínas granulares alfa, los factores de crecimiento y las citoquinas por parte de los megacariocitos, así como las proteasas liberadas por los neutrófilos, inducirían la emperipolesis.

Según Hess et al. (2006), la severidad de la presentación de ehrlichiosis canina dependerá de múltiples factores incluyendo el estado inmune del paciente, la virulencia de la cepa y la presencia de coinfecciones. En ese sentido, De Tomassi et al. (2014) reportaron que los disturbios más frecuentes observados en extendidos de médula ósea de perros infectados o coinfectados con patógenos transmitidos por vectores son la disgranulopoyesis y dismegacariocitopoyesis. Sin embargo, no existen datos bibliográficos que reporten la evidencia de dismegaca- 
riocitopoyesis selectiva secundaria en un perro con ehrlichiosis canina mediante extendidos de Buffy coat.

El hemograma de la paciente presentó una pancitopenia moderada, la cual podría ser atribuida a la ehrlichiosis canina aguda; sin embargo, Nelson y Couto (2014) lo relacionaron más a la fase crónica de la enfermedad como consecuencia de una hipoplasia de la médula ósea. Con relación al conteo de leucocitos, los datos del hemograma muestran una moderada leucopenia, caracterizada por una leve neutropenia y una moderada linfopenia; hallazgos en la cinética de los leucocitos que podrían observarse en la fase aguda de la ehrlichiosis canina según Moreira et al. (2003). Adicionalmente, en el frotis sanguíneo se observó una leve cantidad de linfocitos reactivos y linfocitos atípicos, los cuales han sido relacionados con la presentación de ehrlichiosis canina (Harrus y Waner, 2011).

A través del cálculo de los índices eritrocíticos se identificó anemia moderada caracterizada por ser de tipo microcítica normocrómica; la cual evidenció leve anisocitosis y policromasia. Según Stockam y Scott (2008), la microcitosis eritrocítica suele asociarse con patologías inflamatorias (crónicas) o hepáticas, a través de la alteración en el transporte de hierro exógeno.

Respecto a la serie trombocítica circulante, el valor de 90000 plaquetas por microlitro puede ser catalogado como una trombocitopenia moderada. Así mismo, en los frotis se observó una moderada cantidad de plaquetas hipogranulares y macroplaquetas, características citológicas que estarían reflejando el desarrollo de una trombopoyesis activa. Aunque la trombocitopenia moderada podria ser atribuida a la producción temprana de anticuerpos antiplaquetarios (Waner $e t$ al., 1995), es probable que el desarrollo de la dismegacaricitopoyesis haya contribuido sustancialmente en la disminución del conteo de plaquetas (Weiss, 2004). Se ha reportado que los megacariocitos con núcleos no lobulados a hipolobulados son ineficientes en la producción de plaquetas (Feng et al., 2016).

La identificación de mórulas de Ehrlichia spp se puede realizar en monocitos y linfocitos (Elias, 1991); sin embargo, su presencia en leucocitos se circunscribe a la fase aguda de la enfermedad (Rikihisa, 1991), por lo que se pudo encuadrar el presente caso en dicho periodo de la enfermedad, donde ya se estarían sintetizando los anticuerpos específicos contra E. canis / E. ewingii encontrados a través del Kit SNAP ${ }^{\circledR} 4 \mathrm{Dx}{ }^{\circledR}$ Plus. Aunque es factible la coinfección con $E$. canis y E. ewingii por el tipo de células sanguíneas (monocitos y neutrófilos) donde fueron observadas las mórulas, se podría considerar que la paciente presentó una infección con $E$. canis, donde la variación antigénica de $E$. canis sería la responsable de la presentación atípica de las mórulas en los neutrófilos.

Las anormalidades bioquímicas más frecuentes encontradas en perros infectados con Ehrlichia canis son hipoalbuminemia, hiperglobulinemia e hipergammaglobulinemia (Harrus et al., 1996). En el presente caso, se determinó una leve hipoalbuminemia asociada a una moderada hiperglobulinemia, donde el cálculo de la ratio albuminas/globulinas (0.53) indicó un predominio de las globulinas con relación al rango de referencia (0.6-1.1) descrito por Latimer (2011) para caninos adultos.

El diagnóstico de los SMD se realiza en base a los criterios propuestos por la OMS a través de la evaluación de frotices de sangre periférica y citologías de médula ósea (Swerdlow et al., 2017). Sin embargo, en el presente caso no fue posible el abordaje de la médula ósea del paciente debido a la reminiscencia del dueño a las posibles complicaciones del procedimiento. 


\section{Literatura Citada}

1. de Tommasi AS, Otranto D, Furlanello T, Tasca S, Cantacessi $C$, Breitschwerdt EB, Stanneck D, et al. 2014. Evaluation of blood and bone marrow in selected canine vector-borne diseases. Parasite Vector 7: 534-543. doi: 10.1186/s13071-014-0534-2

2. Elias E. 1992. Diagnosis of ehrlichiosis from the presence of inclusion bodies or morulae of E. canis. J Small Anim Pract 33: 540-543. doi: $10.1111 / \mathrm{j} .1748$ 5827.1992.tb01048.x

3. Feng G, Gale RP, Cui W, Cai W, Huang G, Xu Z, Qin T, et al. 2016. A systematic classification of megakaryocytic dysplasia and its impact on prognosis for patients with myelodysplastic syndromes. Exp Hematol 5: 1-12. doi: 10.1186/s40164-016-0041-6

4. Harrus S, Waner T, Avidar Y, Bogin E, Peh H, Bark H. 1996. Serum protein alterations in canine ehrlichiosis. Vet Parasitol 66: 241-249. doi: 10.1016/ s0304-4017(96)01013-8

5. Harrus S, Waner T. 2011. Diagnosis of canine monocytotropic ehrlichiosis (Ehrlichia canis): an overview. Vet J 187: 292-296. doi: 10.1016/j.tvj1.2010.-02.001

6. Harvey $J W .2012$. Veterinary hematology. A diagnostic guide and color atlas. USA: Elsevier. 360 p.

7. Hess PR, English RV, Hegarty BC, Brown GD, Breitschwerdt EB. 2006. Experimental Ehrlichia canis infection in the dog does not cause immunosuppression. Vet Immunol Immunop 109: 117-125. doi: 10.1016/j.vetimm.2005.07.027

8. Latimer KS. 2011. Duncan \& Prasse's veterinary laboratory medicine: clinical pathology. $5^{\text {th }}$ ed. USA: Wiley-Blackwell. $509 \mathrm{p}$.

9. Moreira SM, Bastos C V, Araújo RB, Santos M, Passos LMF. 2003. Retrospective study (1998-2001) on canine ehrlichiosis in Belo Horizonte, MG
Brazil. Arq Bras Med Vet Zoo 55: 141147. doi: 10.1590/S0102-09352003000200003

10. Nelson RW, Couto CG. 2014. Small animal internal medicine. $5^{\text {th }}$ ed. Canada: Elsevier. $1473 \mathrm{p}$.

11. Rastogi V, Sharma R, Misra SR, Yadav L, Sharma V. 2014. Emperipolesis - a review. J Clin Diag Res 8: 12. doi: 10.7860/JCDR/2014/10361.5299

12. Rikihisa Y. 1991. The tribe Ehrlichieae and ehrlichial diseases. Clin Microbiol Rev 4: 286-308. doi: 10.1128/cmr.4.3.286

13. Schmitt A, Jouault H, Ghichard J. 2000. Pathological interaction between megakaryocytes and polymorphonuclear leukocytes in myelofibrosis. Blood 96: 1342-1347.

14. Stockham SL, Scott MA. 2008. Fundamentals of veterinary clinical pathology. $2^{\text {nd }}$ ed. USA: Blackwell. $924 \mathrm{p}$.

15. Swerdlow SH, Campo E, Harris HL, Jaffe ES, Pileri SA, Stein H, Thiele J. 2017. WHO classification of tumours of haematopoietic and lymphoid tissues. $4^{\text {th }}$ ed. WHO. $588 \mathrm{p}$.

16. Tefferi A, Vardiman JW. 2009. Myelodysplastic syndromes. New Engl J Med 361: 1872-1885.

17. Triantafyllidis I, Ciobanu A, Stanca $O$, Lupu AR. 2012. Prognostic factors in myelodysplastic syndromes. Maedica 7: 295-302.

18. Waner T, Harrus S, Weiss DJ, Bark H, Keysary A. 1995. Demonstration of serum antiplatelet antibodies in experimental acute canine ehrlichiosis. Vet Immunol Immunop 48: 177-182. doi: 10.1016/0165-2427(95)05420-b

19. Weiss DJ, Smith SA. 2000. Primary myelodysplastic syndromes of dogs: a report of 12 cases. J Vet Int Med 14: 491-494. doi: 10.1892/0891-6640(2000)014<0491:pmsoda $>2.3 . c 0 ; 2$

20. Weiss DJ, Aird B. 2001. Cytologic evaluation of primary and secondary myelodysplastic syndromes in the dog. Vet Clin Path 30: 67-75. doi: 10.1111/ j.1939-165X.2001.tb00261.x 
21. Weiss DJ. 2004. Selective dysmegakaryopoiesis in thrombocytopenic dogs (1996-2002). J Comp Clin Path 13: 24-28.

22. Weiss DJ. 2005. Recognition and classification of dysmyelopoiesis in the dog: a review. J Vet Int Med 19: 147-
154. doi: 10.1892/0891-6640(2005)19$<147$ :racodi $>2.0 . c 0 ; 2$

23. Xia P, Wang S, Guo Z, Yao X. 2008. Emperipolesis, entosis and beyond: dance with fate. Cell Res 18: 705-707. doi: 10.7860/JCDR/2014/10361.5299 